\title{
Melphalan Flufenamide: a Peptide-Drug Conjugate for the Treatment of Multiple Myeloma
}

\author{
Dickran Kazandjian and Ola Landgren \\ Myeloma Service, Sylvester Comprehensive Cancer Center, University of Miami, Miami, FL, USA
}

DOI: https://doi.org/10.17925/OHR.2021.17.2.101

M elphalan flufenamide (melflufen) is a peptide-drug conjugate prodrug for melphalan, an alkylating cytotoxic chemotherapy used in the treatment of multiple myeloma. Melflufen recently received accelerated approval from the US Food and Drug Administration (FDA) for the treatment of patients who have received four prior lines of myeloma treatment. The accelerated approval was based on the results of the HORIZON study, which showed an overall response rate of $29 \%$ in the intention-to-treat population with a median duration of response (mDoR) of 5.5 months and 24\% with a mDoR of 4.2 months in the FDA-approved indication subgroup. Adverse events were mostly haematological values. Although these results appear modest, they are in line with what would be expected in a heavily pretreated patient population, and similar to results for other approved drugs. The confirmatory OCEAN trial (a study of melphalan flufenamide [melflufen]-dex or pomalidomide-dex for RRMM patients refractory to lenalidomide) was reported to have met its primary endpoint of improved progression-free survival compared with pomalidomide. However, on 8 July 2021, the FDA published an alert that the melflufen arm in the OCEAN trial was associated with an increased risk of death, and placed melflufen studies on partial hold. While details are currently unknown, an ongoing subgroup analysis is being performed to determine whether certain patient populations benefit more or less in terms of survival. Several other studies are ongoing, and their results are to be determined. Importantly, after discussion with the FDA, melflufen was withdrawn from the US market based on data from the phase III OCEAN study on 22 October 2021. Based on available published data and our clinical experience, we believe melflufen may be a worthy tool in the myeloma physician's armamentarium but until the earlier mortality signal is resolved, it will not, for the most part, be available in the clinic.

\section{Keywords}

Melphalan flufenamide, multiple myeloma, relapsed refractory multiple myeloma, melphalan, US Food and Drug Administration approval

Disclosures: Dickran Kazandjian has participated on an Advisory Board for Bristol Myers Squibb and Arcellx. Ola Landgren has received research funding from Amgen, Celgene, Janssen, Takeda, Glenmark, Seattle Genetics, Karyopharm; honoraria/Advisory Boards from Adaptive, Amgen, Binding Site, BMS, Celgene, Cellectis, Glenmark, Janssen, Juno, Pfizer; and serves on independent datamonitoring committees for Takeda, Merck, Janssen and Theradex.

Acknowledgements: Dickran Kazandjian and Ola Landgren thank the Sylvester Comprehensive Cancer Center (Core Grant P30 CA240139) for supporting this work. Ola Landgren also thanks the Paula and Rodger Riney Foundation for generous support of his research programme.

Review process: Double-blind peer review.

Compliance with ethics: This article involves a review of the literature and did not involve any studies with human or animal subjects performed by any of the authors. Data availability: Data sharing is not applicable to this article as no datasets were generated or analysed during the current study.

Authorship: The named authors meet the International Committee of Medical Journal Editors (ICMJE) criteria for authorship of this manuscript, take responsibility for the integrity of the work as a whole, and have given final approval for the version to be published.

Access: This article is freely accessible at touchONCOLOGY.com (C) Touch Medical Media 2021

Received: 28 July 2021

Accepted: 6 September 2021

Published online: 14 December 2021

Citation: touchREVIEWS in Oncology \& Haematology. 2021;17(2):101-6

Corresponding author: Dickran Kazandjian, Sylvester Comprehensive Cancer Center, 1120 NW 14th Street, Clinical Research Bldg,

Miami, FL 33136, USA. E: dkazandjian@miami.edu

Twitter: @dikokaz

Support: No funding was received in the publication of this article.
Multiple myeloma (MM) is the second most common haematological malignancy, affecting an estimated 450,600 patients worldwide, with an estimated 34,920 new diagnoses and 12,410 patient deaths in the USA in 2021. ${ }^{12}$ Although established curative therapies for MM have not yet been defined, we have observed an incremental increase in survival, with a doubling of 5-year survival rates owing to the advent of novel therapeutics. ${ }^{3.4}$ Based on extrapolations from available population-based databases, it seems reasonable to surmise that a patient with newly diagnosed MM, without underlying comorbidities, will have an average overall survival (OS) of more than 10 years. ${ }^{4}$ The approval of bortezomib, in 2003, represented the first novel therapy to be approved, and since then an additional dozen drugs with various novel mechanisms of action, including immunotherapies, have been approved. ${ }^{5}$ Most recently, the US Food and Drug Administration (FDA) approved the first cellular therapy for myeloma, the chimeric antigen receptor (CAR) T-cell therapy, idecabtagene vicleucel. ${ }^{6}$

Over the last 10 years, the advancement of novel treatments for MM has been astonishing; two decades ago, life expectancy was dismal, given the level of toxicity and relative ineffectiveness of traditional cytotoxic chemotherapy? The first intervention that changed the tide was the development of high-dose melphalan chemotherapy and autologous stem cell support (HDM-ASCT). ${ }^{8}$ Initial trials of HDM were associated with improved efficacy over standard-dose cytotoxic regimens, but at the expense of bone marrow toxicity, leading to delayed recovery time and associated complications. ${ }^{9.10}$ It was ASCT that, in combination with HDM, led to decreased toxicity and improved outcomes, supporting the further development of this approach by Barlogie, McElwain and others in the mid-1980s.11,12 


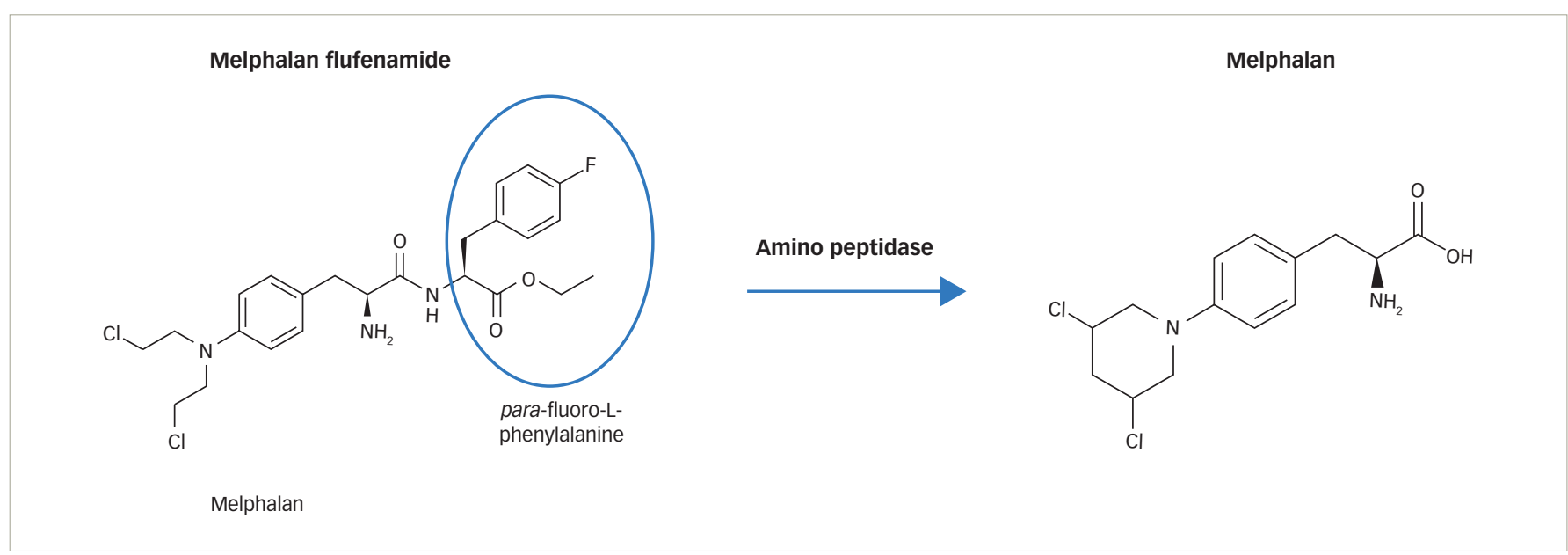

Intracellular aminopeptidases in myeloma cells cleave melphalan flufenamide to the active alkylating drug melphalan (open licence figures). ${ }^{43,44}$

At present, the standard first-line treatment for newly diagnosed MM includes novel proteosome inhibitors and immunomodulatory-drug triplet-based regimens, which have shown effective and sustained minimal residual disease (MRD)-negative remissions. ${ }^{13,14}$ Most recently, clinical trials have added the anti-CD38 monoclonal antibody, daratumumab, on top of novel triplet backbone combinations, yielding high rates of MRD negativity (up to 71\%) and high rates of progressionfree survival (PFS), in the absence of HDM-ASCT. ${ }^{15,16}$ These exponential improvements in efficacy with novel triplet- and quadruplet-based therapies have changed the treatment paradigm for $\mathrm{MM}$, and raised questions regarding the value of first-line HDM-ASCT. ${ }^{17}$ However, in patients who achieve early MRD-negative remission after stem cell harvest, delaying HDM-ASCT until the time of relapse is a favourable approach to reduce both early and delayed toxicity. Therefore, melphalan chemotherapy is likely to remain in the MM physician's treatment toolbox, for now, for various specific indications. As patients with MM live longer and receive subsequent lines of therapy, there remains an unmet need for available drugs in the heavily pretreated, relapsed and refractory MM (RRMM) setting.

The recent development, and ultimate FDA approval, of melphalan flufenamide (melflufen; Pepaxto ${ }^{\oplus}$, Oncopeptides, Inc. Waltham, MA USA) is an example of taking an established active MM chemotherapy (i.e. melphalan) and modifying it to be more targeted with regard to drug delivery. It is a peptide-drug conjugate that, by interacting with aminopeptidases, releases the active alkylating agent specifically into cancer cells, avoiding normal cells. In our opinion, melflufen is a respectable addition to the MM clinician's armamentarium in the treatment of patients with RRMM requiring multiple subsequent lines of therapy. In this review, we summarize the biological rationale, preclinical studies, early-phase clinical studies and the pivotal trials leading to the approval of melflufen for the treatment of RRMM, and describe ongoing ancillary and supportive clinical studies.

\section{Preclinical overview of melflufen}

Melflufen consists of melphalan conjugated to the peptide para-fluoroL-phenylalanine, creating a lipophilic drug (Figure 1). ${ }^{18}$ Once melflufen enters myeloma cells, cytotoxic activity is dependent on the cell's aminopeptidase $\mathrm{N}$ activity to cleave the active alkylating agent. The intracellular accumulation of melflufen is thought to occur rapidly over the cell membrane by lipophilic passive diffusion. Once the prodrug has accumulated intracellularly, the formation of the active drug is dependent on high expression of aminopeptidases, which have been observed in MM and other malignant histologies. ${ }^{18}$ Therefore, the antitumour activity of melflufen is less dependent on exposure time and half-life than melphalan, and in vitro, peak activity is observed after just 30 minutes.

In both in vitro and in vivo model systems, melflufen has been shown to be more potent than melphalan, possibly deploying a more efficient therapeutic strategy for delivering higher concentrations of intracellular melphalan, and in human plasmacytoma xenograft mouse models, treatment with melflufen significantly inhibited MM tumour growth and prolonged survival compared with melphalan. ${ }^{19,20}$ With regard to toxicity, transient haematological toxicity and end-organ toxicities of the testes, epididymides, bone marrow and spleen were observed in animal studies. ${ }^{21}$

\section{Early-phase clinical studies}

The first-in-human phase I/lla study of melflufen was in patients with advanced solid tumours and no available treatment options. ${ }^{22}$ Once the initial safety profile was characterized, melflufen was evaluated in the 0-12-M1 study, a single-arm, multicentre, dose-expansion and confirmation phase I/II study in patients with RRMM who had received at least two prior lines of therapy. ${ }^{23}$ In phase I, a maximum tolerated dose of melflufen $40 \mathrm{mg}$ every 21 days plus weekly dexamethasone was established; this was extended to a 28-day cycle in phase II to prolong haematological recovery time. Of the 45 patients who received this combination in phase $I$, the overall response rate (ORR) was 31\%, with nine patients achieving a partial response (PR) or better with a median duration of response (DoR) of 8.4 months. Commonly reported adverse events (AEs) included thrombocytopenia, neutropenia, anaemia, pyrexia, asthenia, fatigue, nausea and diarrhoea.

\section{Pivotal trials and FDA approval The HORIZON study}

On 26 February 2021, the FDA granted accelerated approval to melflufen in combination with dexamethasone for patients with RRMM who have received at least four prior lines of therapy and whose disease is refractory to at least one proteasome inhibitor, one immunomodulatory agent and one CD38-directed monoclonal antibody, based on the results of the HORIZON study (OP-106; NCT02963493). ${ }^{24,25}$ The HORIZON study was a single-arm, multicentre, phase II study in patients with RRMM refractory to pomalidomide and/or an anti-CD38 monoclonal antibody. ${ }^{26}$ Eligible adult patients had an Eastern Cooperative Oncology Group performance status (ECOG PS) of 0-2, RRMM with disease progression and measurable disease, 
Table 1: HORIZON study efficacy results as assessed by study investigators ${ }^{26}$ and subgroup data ${ }^{27}$

\begin{tabular}{|c|c|c|c|}
\hline \multirow[t]{2}{*}{ Response } & \multicolumn{3}{|c|}{ Melflufen with dexamethasone } \\
\hline & $\begin{array}{l}\text { All patients }{ }^{26} \\
(\mathrm{~N}=157)\end{array}$ & $\begin{array}{l}\text { Triple-class refractory }{ }^{26} \\
(n=119)\end{array}$ & $\begin{array}{l}\text { FDA Subgroup analysis with } \geq 4 \text { prior } \\
\text { lines of therapy }{ }^{27} \\
(\mathrm{n}=97)\end{array}$ \\
\hline Overall response rate, \% (95\% Cl) & $29(22-37)$ & $26(18-35)$ & $23.7(15.7-33.4)$ \\
\hline Stringent complete response, \% & 1 & 0 & 0 \\
\hline Complete response, \% & 0 & 0 & 0 \\
\hline Very good partial response, $\%$ & 11 & 11 & 9.3 \\
\hline Partial response, \% & 18 & 15 & 14.4 \\
\hline Median duration of response, months $(95 \% \mathrm{Cl})$ & $5.5(3.9-7.6)$ & $4.4(3.4-7.6)$ & $4.2(3.2-7.6)$ \\
\hline
\end{tabular}

$\mathrm{Cl}=$ confidence interval; FDA = US Food and Drug Administration.

and had received $\geq 2$ prior lines of therapy (including an immunomodulatory agent and proteasome inhibitor, and were refractory to pomalidomide and/or an anti-CD38 monoclonal antibody). Patients received melflufen $40 \mathrm{mg}$ as a 30-minute central intravenous infusion on day 1 of each 28day cycle in combination with dexamethasone $40 \mathrm{mg}$ oral weekly until disease progression or unacceptable toxicity, or at the discretion of the physician/patient. The primary endpoint was ORR, per the International Myeloma Working Group response criteria, as assessed by the investigator and confirmed by an independent review committee (IRC). Secondary endpoints included DoR, PFS, OS and safety. The study was designed to enrol 150 patients with the statistical hypothesis that assumed an ORR of $30 \%$ with an exact $95 \%$ confidence interval (Cl) of $23-38 \% .{ }^{26}$

At the data cut-off date, $83 \%$ of the 157 study-treated patients had discontinued treatment mostly due to disease progression (56\%) or AEs (17\%). The median duration of treatment was 3.8 months. Patients had a median age of 65 years and received a median of five prior lines, $98 \%$ were refractory to their last line of therapy, $76 \%$ had triple-class refractory disease, 38\% had high-risk cytogenetics, and 25\% had an International Staging System stage III disease. ${ }^{26}$

The HORIZON study reported an ORR per investigator assessment of 29\% (95\% $\mathrm{Cl} 22-37 \%)$, a median time to response of 1.9 months and a median DoR of 5.5 months ( $95 \% \mathrm{Cl}$ 3.9-7.6 months), with one patient achieving a stringent complete response (CR), 17 a very good PR (VGPR) and 28 a PR. In the triple-class refractory subgroup, the ORR was $26 \%(95 \% \mathrm{Cl}$ 18-35\%) and median DoR was 4.4 months (95\% Cl 3.4-7.6 months), with 13 patients achieving a VGPR and 18 a PR. The ORR per the IRC was $30 \%$ overall ( $95 \% \mathrm{Cl} 23-38 \%$ ) and $26 \%$ in the triple-class refractory subgroup (95\% Cl 18-35\%). Median PFS was 4.2 months (95\% Cl 3.4-4.9 months) overall and 3.9 months ( $95 \% \mathrm{Cl} 3.0-4.6$ months) in the tripleclass refractory subgroup. Median OS was 11.6 months (95\% Cl 9.3-15.4 months) overall and 11.2 months ( $95 \% \mathrm{Cl}$ 7.7-13.2 months) in the tripleclass refractory subgroup (Table 1). ${ }^{26}$

Treatment-emergent AEs of any grade were observed in all patients, with common non-haematological AEs being nausea (32\%), fatigue (29\%), asthenia (27\%), diarrhoea (27\%), pyrexia (24\%), cough (17\%), upper respiratory tract infection (16\%), constipation (15\%), decreased appetite (14\%), hypokalaemia (14\%), peripheral oedema (14\%), headache (13\%), vomiting (13\%), bone pain (13\%), pain in extremity (13\%) and pneumonia (13\%). Grade $\geq 3$ AEs occurred in 150 patients (96\%), with the most common being neutropenia (79\%), thrombocytopenia (76\%), anaemia (43\%), pneumonia (10\%) and hypophosphataemia (5\%). Serious AES occurred in 77 patients (49\%), most common of which were pneumonia (9\%) and febrile neutropenia (5\%) (Table 2). ${ }^{26}$
The results from the pivotal HORIZON study were the basis for the submission of a new drug-licensing application for registration to the FDA, which granted melflufen priority review status and a Prescription Drug User Fee Act action goal date of 28 February 2021..2 Melflufen was granted accelerated approval based on determining a favourable benefit-risk profile. In the FDA executive review, approval was based on the ORR and DoR from a subgroup analysis of 97 patients in the HORIZON study who received $\geq 4$ prior lines of therapies and were refractory to a proteasome inhibitor, immunomodulatory drug and an anti-CD38 monoclonal antibody. The ORR per investigator assessment in this subgroup was $23.7 \%$ (95\% Cl 15.7-33.4\%), and the median DoR was 4.2 months ( $95 \% \mathrm{Cl} 3.2-7.6$ months) (Table 1).

In terms of safety, the FDA Prescribing Information describes the following adverse reactions: thrombocytopenia (grade 3,26\%; grade 4, 54\%), neutropenia (grade 3, 41\%; grade 4, 40\%; febrile neutropenia, $6 \%$ ), anaemia (grade 3,50\%), infection (grade 3, 20\%; grade 4, $1.9 \%$; fatal infection, $<1 \%$, increased risk of mortality with dosages higher than the recommended dosage, secondary malignancies (myelodysplastic syndromes or acute leukaemia) and embryo-foetal toxicity. ${ }^{27}$

\section{The OCEAN study}

Due to the lack of a randomized control arm in the HORIZON study, FDA accelerated approval was based on a surrogate endpoint (ORR) thought to predict clinical benefit in treating a serious disease with unmet medical need (RRMM), as is standard protocol. ${ }^{28}$ However, accelerated approval requires the sponsor to confirm clinical benefit by submitting a final study report and dataset from a randomized phase III trial, which, in this case, should verify the clinical benefit of melflufen in patients with RRMM. Patients in said trial should be randomized to receive either melflufen or standard therapy. The primary endpoint should be PFS assessed by an IRC, and secondary endpoints should include ORR and OS. ${ }^{21}$

Final report submission to the FDA is scheduled for February 2022, and will comprise the ongoing, confirmatory, phase III, randomized OCEAN trial (OP-103; NCT03151811), in 495 patients who have received 2-4 prior therapies and are refractory to lenalidomide. Patients have been randomized to receive dexamethasone in combination with either melflufen or pomalidomide. The primary endpoint is PFS, and secondary endpoints are ORR, DOR and OS. ${ }^{25,29}$ However, on 8 July 2021, the FDA requested a partial clinical hold on all melflufen studies, based on updated results from the OCEAN study following an IRC reassessment, which was missing clinical data on 29 patients. ${ }^{30}$ In the new analysis, melflufen met the primary endpoint of superior PFS compared with pomalidomide (hazard ratio [HR] 0.79; 95\% $\mathrm{Cl} 0.64-0.98 ; \mathrm{p}=0.0311$ ) as determined by the IRC. However, the key secondary endpoint of OS had an HR of $1.10(95 \% \mathrm{Cl}$ 
Table 2: Adverse reactions occurring in $\geq 10 \%$ of patients receiving melflufen with dexamethasone from the HORIZON study, as reported in the Prescribing Information ${ }^{27}$

\begin{tabular}{|c|c|c|}
\hline Adverse reaction & All grades (\%) & Grade 3 or $4(\%)$ \\
\hline \multicolumn{3}{|c|}{ General disorders and administration-site disorders } \\
\hline Fatigue & 55 & 6 \\
\hline Pyrexia & 24 & 1.9 \\
\hline Oedema peripheral & 14 & 1.3 \\
\hline \multicolumn{3}{|l|}{ Gastrointestinal disorders } \\
\hline Nausea & 32 & 0.6 \\
\hline Diarrhoea & 27 & 0 \\
\hline Constipation & 15 & 0.6 \\
\hline Vomiting & 13 & 0 \\
\hline \multicolumn{3}{|l|}{ Infections } \\
\hline Respiratory tract infection & 24 & 5 \\
\hline Pneumonia & 13 & 11 \\
\hline \multicolumn{3}{|c|}{ Respiratory, thoracic and mediastinal disorders } \\
\hline Cough & 17 & 0 \\
\hline Dyspnoea & 11 & 1.3 \\
\hline Dyspnoea exertional & 10 & 0 \\
\hline \multicolumn{3}{|c|}{ Metabolism and nutrition disorders } \\
\hline Decreased appetite & 14 & 0.6 \\
\hline Hypokalaemia & 14 & 1.3 \\
\hline Hypocalcaemia & 10 & 0.6 \\
\hline \multicolumn{3}{|l|}{ Nervous system disorders } \\
\hline Headache & 13 & 0 \\
\hline Dizziness & 11 & 0 \\
\hline \multicolumn{3}{|c|}{ Musculoskeletal and connective-tissue disorders } \\
\hline Bone pain & 13 & 1.9 \\
\hline Pain in extremity & 13 & 1.9 \\
\hline Back pain & 12 & 0.6 \\
\hline Arthralgia & 10 & 0 \\
\hline \multicolumn{3}{|l|}{ Psychiatric disorders } \\
\hline Insomnia & 11 & 0.6 \\
\hline \multicolumn{3}{|l|}{ Laboratory abnormalities } \\
\hline Leukocytes decrease & 99 & 88 \\
\hline Platelets decrease & 99 & 80 \\
\hline Lymphocytes decrease & 97 & 95 \\
\hline Neutrophils decrease & 95 & 82 \\
\hline Haemoglobin decrease & 84 & 50 \\
\hline Creatinine increase & 68 & 1 \\
\hline
\end{tabular}

0.85-1.44) in favour of pomalidomide for the intention-to-treat population. Further investigation is ongoing and will include analyses of subgroups that might have driven the detrimental OS. On 22 October 2021, however, Oncopeptides, the manufacturer of melflufen, after discussion with the FDA, withdrew the product from the US market. Therefore, it is no long available to the general MM population, but patients who were previously receiving it and deriving benefit will be able to continue treatment through a compassionate use program with oncopeptides.

\section{Other clinical trials evaluating melflufen \\ Melflufen triplet combinations}

The ongoing, two-arm, phase I/II ANCHOR study (OP-104; NCTO3481556) is evaluating triplet regimens of melflufen ( 30 or $40 \mathrm{mg}$ ) plus dexamethasone in combination with either bortezomib or daratumumab in patients with RRMM, who have received 1-4 prior lines of therapy, and are refractory to an immunomodulatory drug and/or a proteosome inhibitor. ${ }^{31,32}$ In preliminary results, amongst 33 patients in the daratumumab arm, ORR was $70 \%$ ( 1 stringent CR, 1 CR and 10 VGPR), with a median DoR of 12.5 months, and a median PFS of 11.5 months. ${ }^{32}$ In the bortezomib arm ( $\left.n=13\right)$, ORR was $62 \%$, with $1 \mathrm{CR}$ and 4 VGPR. ${ }^{32}$ Furthermore, the daratumumab triplet combination is being compared with daratumumab alone in the phase III, randomized LIGHTHOUSE trial (OP-108; NCT04649060), in patients with RRMM previously treated with an immunomodulatory drug and a proteasome inhibitor. Patient enrolment has begun, with 240 patients planned to be enrolled; the primary endpoint is PFS and key secondary endpoints are ORR, DOR and OS. ${ }^{33,34}$

\section{Clinical pharmacology trials}

The ongoing, phase II BRIDGE study (OP-107; NCT03639610) is evaluating the pharmacokinetics of melphalan during treatment with dexamethasone in patients with RRMM and moderate to severely impaired renal function. ${ }^{35,36}$ Renal and hepatic insufficiency are not expected to alter pharmacokinetics significantly, given that the metabolism of melflufen is dependent on cellular aminopeptidases and melphalan is metabolized by spontaneous hydrolysis in the blood..$^{35}$ The study is ongoing, with seven of the 35 planned patients thus far enrolled. ${ }^{27,37}$

The current approval of melflufen is based on administration by central intravenous infusion, which makes it less practical than standard peripheral intravenous infusions for patients. The PORT study (NCT04412707), a randomized, two-period, cross-over, phase II study, has not yet been activated, but will be recruiting up to 27 patients to compare the pharmacokinetics, safety and tolerability of peripheral versus central intravenous administration of melflufen. ${ }^{38}$ Finally, in the phase I/II ASCENT study (NCT04115956), 46 patients with immunoglobulin light-chain amyloidosis following one line of therapy will be enrolled to evaluate the safety and efficacy of up to eight cycles of melflufen with dexamethasone. ${ }^{39}$ Table 3 summarizes the melflufen drug development pipeline.

\section{Conclusions and future perspectives}

The melflufen drug development programme is a prime example of how an older cytotoxic drug is not just recycled in the context of a changing field towards novel immunotherapies, but also improved by making it more targeted and introducing as a later line of therapy. As triplet and now quadruplet novel therapy combinations have dominated the treatment of newly diagnosed MM, achieving high rates of MRD-negative remission, HDM-ASCT is moving from an early treatment modality to one that is used in the relapsed setting. This is critical, as independent investigators are now showing the detrimental, long-term, mutagenic effects of HDM, and long-term risk of developing secondary cancers. ${ }^{40,41}$ The development of melflufen for later-line treatment places melphalan in a more clinically and biologically rational space in the MM treatment paradigm, where patients have exhausted most of the available treatments. Although, initially, we believed that the later line use of this melphalan prodrug would have a favourable benefit to risk profile, the detrimental survival signal has led us to rethink this balance. Regardless, since the product has been withdrawn from the market, except for rare cases, it is not available for general use. In this practice, melflufen had reasonable tolerability, with toxicities being mostly haematological and treatable. Without experiencing increased deaths anecdotally, the OCEAN trial preliminary data and warning is strong enough for us to have terminated its use.

There are still several uncertainties regarding melflufen. First, is whether the current indication in a heavily pretreated population is the best 
Table 3: Clinical trials for the development and marketing of melflufen

\begin{tabular}{|c|c|c|c|c|c|c|c|c|c|c|}
\hline Study & Indication & $\begin{array}{l}\text { Line of } \\
\text { therapy }\end{array}$ & Trial design & Primary endpoint & $\begin{array}{l}\text { Patients, } \\
\text { N }\end{array}$ & Enrolment* & $\begin{array}{l}\text { Study } \\
\text { start } \\
\text { date }\end{array}$ & $\begin{array}{l}\text { Estimated } \\
\text { primary } \\
\text { endpoint } \\
\text { date }\end{array}$ & $\begin{array}{l}\text { Estimated } \\
\text { study } \\
\text { completion } \\
\text { date }\end{array}$ & Notes \\
\hline $\mathrm{FIH}$ of melflufen ${ }^{22}$ & $\begin{array}{l}\text { Solid } \\
\text { tumours }\end{array}$ & $\begin{array}{l}\text { Exhausted } \\
\text { available } \\
\text { therapy }\end{array}$ & Phase I/lla & $\begin{array}{l}\text { Determine RP2D/ } \\
\text { response }\end{array}$ & 45 & Complete & $\mathrm{n} / \mathrm{a}$ & $\mathrm{n} / \mathrm{a}$ & $\mathrm{n} / \mathrm{a}$ & $\begin{array}{l}\text { In Sweden, } \\
\text { Russia }\end{array}$ \\
\hline $\begin{array}{l}\text { O-12-M1 } \\
(\text { NCT01897714) })^{23}\end{array}$ & RRMM & $\begin{array}{l}\geq 2 \text { lines (inc. } \\
\text { LEN, BTZ) }\end{array}$ & Phase I/Ila & $\begin{array}{l}\text { Determine RP2D/ } \\
\text { ORR }\end{array}$ & $\begin{array}{l}81 \\
\text { (actual) }\end{array}$ & Complete & 7/2013 & $12 / 2017$ & $3 / 2020$ & \\
\hline $\begin{array}{l}\text { HORIZON, OP-106 } \\
\text { (NCT02963493) }^{42}\end{array}$ & $\begin{array}{l}\text { RRMM (POM } \\
\pm \text { anti-CD38 } \\
\text { refractory) }\end{array}$ & $\begin{array}{l}\geq 2 \text { lines (inc. } \\
\text { IMiD, PI) }\end{array}$ & $\begin{array}{l}\text { Phase II, } \\
\text { single-arm, } \\
\text { pivotal }\end{array}$ & ORR & $\begin{array}{l}157 \\
\text { (actual) }\end{array}$ & $\begin{array}{l}\text { Active, not } \\
\text { recruiting }\end{array}$ & $12 / 2016$ & 9/2020 & 9/2022 & $\begin{array}{l}\text { Accelerated } \\
\text { approval } \\
\text { registration } \\
\text { study }\end{array}$ \\
\hline $\begin{array}{l}\text { OCEAN, OP-103 } \\
\left(\text { NCT03151811) }{ }^{29}\right.\end{array}$ & $\begin{array}{l}\text { RRMM (LEN } \\
\text { refractory) }\end{array}$ & $\begin{array}{l}\text { 2-4 lines (inc. } \\
\text { LEN, PI) }\end{array}$ & $\begin{array}{l}\text { Phase III, } \\
\text { randomized } \\
\text { melflu or } \\
\text { POM }\end{array}$ & PFS & 495 & $\begin{array}{l}\text { Active, not } \\
\text { recruiting }\end{array}$ & 6/2017 & $3 / 2021$ & $7 / 2022$ & $\begin{array}{l}\text { Confirmatory } \\
\text { registrational } \\
\text { study }\end{array}$ \\
\hline $\begin{array}{l}\text { ANCHOR, OP-104 } \\
(\text { NCT03481556) } 31,32\end{array}$ & $\begin{array}{l}\text { RRMM (BTZ } \\
\text { refractory) }\end{array}$ & 1-4 lines & $\begin{array}{l}\text { Phase I/Ila, } \\
\text { 2-cohort, } \\
\text { melflu, DEX + } \\
\text { dara or BTZ }\end{array}$ & Toxicity/ORR & 80 & $\begin{array}{l}\text { Active, not } \\
\text { recruiting }\end{array}$ & $4 / 2018$ & $12 / 2021$ & $12 / 2023$ & \\
\hline $\begin{array}{l}\text { LIGHTHOUSE, OP-108 } \\
\text { (NCT04649060) }\end{array}$ & $\begin{array}{l}\text { RRMM (IMiD, } \\
\text { PI refractory) }\end{array}$ & $\begin{array}{l}\geq 3 \text { lines (inc. } \\
\text { IMiD, PI) }\end{array}$ & $\begin{array}{l}\text { Phase III, } \\
\text { randomized, } \\
\text { dara } \pm \text { melflu, } \\
\text { DEX }\end{array}$ & PFS & 240 & $\begin{array}{l}\text { Active, not } \\
\text { recruiting }\end{array}$ & $12 / 2020$ & 6/2023 & $11 / 2025$ & \\
\hline $\begin{array}{l}\text { BRIDGE, OP-107 } \\
\left(^{(N C T 03639610)^{35}}\right.\end{array}$ & $\begin{array}{l}\text { RRMM with } \\
\text { renal insuff }^{+}\end{array}$ & 2-4 lines & $\begin{array}{l}\text { Phase II, } \\
\text { single-arm, } \\
\text { 2-cohort }\end{array}$ & Pharmacokinetics & 35 & $\begin{array}{l}\text { Active, not } \\
\text { recruiting }\end{array}$ & $8 / 2018$ & $12 / 2021$ & 9/2022 & \\
\hline $\begin{array}{l}\text { PORT } \\
(\text { NCT04412707) })^{38}\end{array}$ & $\begin{array}{l}\text { RRMM (IMiD, } \\
\text { PI refractory) }\end{array}$ & $\geq 2$ lines & $\begin{array}{l}\text { Phase II, } \\
\text { randomized, } \\
\text { crossover, } \\
\text { PIV or CVC }\end{array}$ & Pharmacokinetics & 27 & $\begin{array}{l}\text { Active, not } \\
\text { recruiting }\end{array}$ & $8 / 2020$ & $6 / 2021$ & $3 / 2022$ & \\
\hline $\begin{array}{l}\text { ASCENT } \\
(\text { NCT04115956) }\end{array}$ & $\begin{array}{l}\mathrm{AL} \\
\text { amyloidosis }\end{array}$ & $\geq 1$ line & Phase I/II & Toxicity, RP2D/ORR & 46 & $\begin{array}{l}\text { Active, not } \\
\text { recruiting }\end{array}$ & $8 / 2020$ & $2 / 2023$ & $4 / 2024$ & \\
\hline
\end{tabular}

*On 8 July 2021, the US Food and Drug Administration placed all melflufen studies on hold after it was determined that although the primary endpoint of PFS was met, the HR for OS was detrimental for melflufen. 'Estimated glomerular filtration rate: cohort 1, 30-<45 mL/min; cohort 2, 15-<30 mL/min.

$A L=$ immunoglobulin light chain; $B T Z$ = bortezomib; $C V C=$ central venous catheter; dara = daratumumab; $D E X=$ dexamethasone; FIH = first in human; HR = hazard ratio; IMiD = immunomodulatory drug; inc. = including; insuff = insufficiency; $n / a=$ not available; $L E N=$ lenalidomide; melflu = melflufen; ORR = Overall response rate; OS = Overall survival; $P F S=$ progression-free survival; $P I=$ proteosome inhibitor; $P I V=$ peripheral intravenous catheter; $P O M=$ pomalidomide; $R P 2 D=$ recommended phase $/ /$ dose; $R R M M=$ relapsed and refractory multiple myeloma.

use of melflufen, or whether there are synergistic combinations in less heavily treated populations that will prove better. Second, is regarding the suboptimal administration of melflufen, which currently requires central venous catheterization. The results of the PORT study are eagerly awaited in this context. ${ }^{38}$ Finally, it is unknown whether melflufen can be used in the high-dose setting with stem-cell rescue to replace traditional melphalan. Unfortunately, given the recent withdrawal of melflufen from the market, the above questions may never be answered as Oncopeptides has strategically changed the company's direction. We are hopeful that in the near future, the void created will be filled by more tolerable and efficacious therapies as there is still a significant unmet need in RRMM. (https://www.oncopeptides.com/en/media/ press-releases/oncopeptides-withdraws-pepaxto-in-us-scale-downorganization-and-focus-on-rd) $\square$
1. World Health Organization, International Agency for Research on Cancer, Cancer Today. Available at: https://gco.iarc.fr/today/ home (accessed 20 June 2021).

2. Siegel RL, Miller KD, Fuchs HE, Jemal A. Cancer statistics, 2021. CA Cancer J Clin. 2021;71:7-33.

3. Kazandjian D, Landgren O. A look backward and forward in the regulatory and treatment history of multiple myeloma: approval of novel-novel agents, new drug development, and longer patient survival. Semin Oncol. 2016;43:682-9.

4. National Cancer Institute. Surveillance, Epidemiology, and End Results Program, Myeloma: Recent Trends in SEER Relative Survival Rates, 2000-2018. Available at: https://seer.cancer. Survival Rates, 2000-2018. Available at: $h$ ttps://seer.cancer.
gov/explorer/application.html?site=89\&data_type $=4 \& g r a p h$ type $=2 \&$ compareBy=sex\&chk_sex_1 1 1\&chk_sex_3 $3 \&$ chk_ sex $\_2=2 \&$ race $=1$ \&age_range $=1$ \&stage $=101 \&$ survival $\_$ interval $=5 \&$ advopt_precision $=1$ \&advopt_show_ci=on\&advopt display $=1$ (accessed 15 June 2021).

5. US Food and Drug Administration. VELCADE® (bortezomib): prescribing information. Available at: www.accessdata.fda.gov/ drugsatfda_docs/label/2014/021602s040lbl.pdf (accessed 14 October 2021).

6. Shah UA, Mailankody S. CAR T and CAR NK cells in multiple myeloma: expanding the targets. Best Pract Res Clin Haematol myeloma: expan

7. Kazandjian D. Multiple myeloma epidemiology and survival: A Kazandjian D. Multiple myeloma epidemiology and

unique malignancy. Semin Oncol. 2016;43:676-81.
Kazandjian D, Landgren O. Delaying the use of high-dose melphalan with stem cell rescue in multiple myeloma is ready for prime time. Clin Adv Hematol Oncol. 2019;17:559-68. 9. Selby PJ, McElwain TJ, Nandi AC, et al. Multiple myeloma treated with high dose intravenous melphalan. Br J Haematol. 1987;66:55-62.

10. Kazandjian D, Dew A, Hill E. The changing role of high dose melphalan with stem cell rescue in the treatment of newly diagnosed multiple myeloma in the era of modern therapies - back to the futurel Best Pract Res Clin Haem 2020;33:101150.

11. Barlogie B, Hall R, Zander A, et al. High-dose melphalan with autologous bone marrow transplantation for multiple myeloma. autologous bone marrow

12. MCElwain TJ, Powles RL. High-dose intravenous melphalan for plasma-cell leukaemia and myeloma. Lancet. 1983;2(8354):822-4. 
13. Kazandjian D, Korde N, Mailankody S, et al. Remission and progression-free survival in patients with newly diagnosed multiple myeloma treated with carfitzomib, lenalidomide and multiple myeloma treated wh car lzomib, Icnalidomide, and dexamethasone: five-year foll

14. Perrot $A$, Lauwers-Cances $V$, Corre J, et al. Minimal residual disease negativity using deep sequencing is a major prognostic factor in multiple myeloma. Blood. 2018;132:2456-64.

5. Landgren O, Hultcrantz M, Diamond B, et al. Safety and effectiveness of weekly carfilzomib, lenalidomide, dexamethasone, and daratumumab combination therapy for patients with newly diagnosed multiple myeloma: the MANHATTAN nonrandomized clinical trial. JAMA Oncol. 2021;7:862-8

16. Voorhees PM, Kaufman JL, Laubach J, et al. Daratumumab, lenalidomide, bortezomib, and dexamethasone for transplanteligible newly diagnosed multiple myeloma: the GRIFFIN trial. Blood 2020:136:936-45.

17. Kazandjian D, Mo CC, Landgren O, Richardson PG. The role of high-dose melphalan with autologous stem-cell transplan in multiple myeloma: is it time for a paradigm shift? $\mathrm{Br}\rfloor$ Haematol. 2020:191:692-703.

18. Wickström $M$, Nygren $P$, Larsson $R$, et al. Melflufen - a peptidase-potentiated alkylating agent in clinical trials. Oncotarget. 2017;8:66641-55

19. Gullbo J, Lindhagen E, Bashir-Hassan S, et al. Antitumor efficacy and acute toxicity of the novel dipeptide melphalanyl-p-Lfluorophenylalanine ethyl ester (J1) in vivo. Investig New Drugs. 2004;22:411-20.

20. Chauhan D, Ray A, Viktorsson $\mathrm{K}$, et al. In vitro and in vivo antitumor activity of a novel alkylating agent, melphalanflufenamide, against multiple myeloma cells. Clin Cancer Res. 2013;19:3019-31

21. FDA. Pepaxto Multi-Discipline Review; Drugs@FDA: FDAApproved Drugs, NDA 214383 (accessed 16 July 2021).

22. Berglund Å, Ullén A, Lisyanskaya A, et al. First-in-human phase $1 /$ /la clinical study of the peptidase potentiated alkylator melflufen administered every three weeks to patients with advanced solid tumor malignancies. Investig New Drugs. 2015;33:1232-4

23. Richardson $P G$, Bringhen $S$, Voorhees $P$, et al. Melflufen plus dexamethasone in relapsed and refractory multiple myelom (O-12-M1): a multicentre, international, open-label, phase 1-2 study. Lancet Haematol. 2020;7:e395-407.

24. US Food and Drug Administration. FDA grants accelerated approval to melphalan flufenamide for relapsed or refractory multiple myeloma. 2021. Available at: www.fda.gov/drugs/ resources-information-approved-drugs/fda-grants-accelerated approval-melphalan-flufenamide-relapsed-or-refractorymultiple-myeloma (accessed 14 October 2021).

25. Dhillon S. Melphalan flufenamide (melflufen): first approval. Drugs. 2021;81:963-9.

26. Richardson PG, Oriol A, Larocca A, et al. Melflufen and dexamethasone in heavily pretreated relapsed and refractory multiple myeloma. J Clin Oncol. 2021;39:757-67.

27. US Food and Drug Administration. PEPAXTO ${ }^{\oplus}$ (melphalan flufenamide) prescribing information. 2021. Available at: www.accessdata.fda.gov/drugsatfda_docs/ label/2021/214383s00olbl.pdf (accessed 13 October 2021).

28. US Food and Drug Administration. Accelerated Approval Program. Available at: www.fda.gov/drugs/information-healthcare-professionals-drugs/accelerated-approval-program (accessed 14 October 2021)

29. ClinicalTrials.gov. A Study of Melphalan Flufenamide (Melflufen)-Dex or Pomalidomide-dex for RRMM Patients Refractory to Lenalidomide (OCEAN). ClinicalTrials.gov Identifie NCT03151811. Available at: https://clinicaltrials.gov/ct2/show/ NCT03151811 (accessed 14 October 2021).

30. Oncopeptides. Updated results from phase 3 OCEAN study shows melflufen met primary endpoint of superior PFS overall survival data lead to partial clinical hold. 2021. Available at: www.oncopeptides.com/en/media/press-releases/updatedresults-from-phase-3-ocean-study-shows-melflufen-metprimary-endpoint-of-superior-pfs--overall-survival-data-lead-topartial-clinical-hold (accessed 14 October 2021)

31. Ocio EM, Efebera YA, Hájek R, et al. ANCHOR (OP-104): Melflufen plus dexamethasone (dex) and daratumumab (dara) or bortezomib (BTZ) in relapsed/refractory multiple myeloma (RRMM) refractory to an IMiD and/or a proteasome inhibitor (P) (RRMM)

2. Hajek R, POur L, Granell M, et al.ANCHOR (OP-104): Melfufen plus dexamethasone (dex) and bortezomib (BTZ) in relapsed/ refractory multiple myelona (RRMM) - optimal dose, upda efficacy and safe 15):8037-8037

33. Mateos M-V, Pour L, Ocio EM, et al. LIGHTHOUSE (OP08): A phase 3 study of melflufen in combination with dexamethasone (dex) and daratumumab (dara) versus dara in relapsed/refractory multiple myeloma (RRMM) patients (pts). J Clin Oncol. 2021:39(Suppl. 15):TPS8051-TPS8051.
34. ClinicalTrials.gov. Study of Melphalan Flufenamide (Melflufen) in Combination With Daratumumab in Relapsed Refractory Multiple Myeloma (LIGHTHOUSE). ClinicalTrials.gov Identifier: NCT04649060. Available at: https://clinicaltrials.gov/ct2/show/ NCT04649060 A (accessed 14 October 2021).

35. Mateos M-V, Bladé J, Bringhen S, et al. Melflufen: a peptidedrug conjugate for the treatment of multiple myeloma. J Clin Med. 2020;9:3120.

36. ClinicalTrials.gov. A PK Study of Melphalan During Treatment with Melphalan Flufenamide (Melflufen) and Dex in RRMM Pat with Impaired Renal Function. ClinicalTrials.gov Identifier. NCT03639610. Available at: https://clinicaltrials.gov/ct2/show/ NCT03639610 (accessed 14 October 2021).

37. ClinicalTrials.gov. A PK Study of Melphalan During Treatment with Melphalan Flufenamide (Melflufen) and Dex in RRMM Pat with Impaired Renal Function. ClinicalTrials.gov Identifier: NCT03639610. Available at: https://clinicaltrials. gov/ct2/show/ NCT03639610 (accessed 14 October 2021).

38. ClinicalTrials.gov. A PK, Safety and Tolerability Study of Peripheral and Central Infusion of Melflufen in RRMM Patients (PORT). ClinicalTrials.gov Identifier: NCT04412707. Available at: https://clinicaltrials. gov/ct2/show/NCT04412707 (accessed 14 October 2021).

39. ClinicalTrials.gov. A Clinical Study of Melphalan Flufenamide (Melflufen) and Dexamethasone for Patients with Immunoglobulin Light Chain (AL) Amyloidosis. ClinicalTrials.gov Identifier: NCT04115956. Available at: https://clinicaltrials.gov/ ct2/show/NCT04115956 (accessed 14 October 2021).

40. Maura F, Weinhold N, Diamond B, et al. The mutagenic impact of melphalan in multiple myeloma. Leukemia. 2021;35:2145-50.

41. Samur MK, Roncador M, Aktas-Samur A, et al. High-dose melphalan significantly increases mutational burden in multiple myeloma cells at relapse: results from a randomized study in multiple myeloma. Blood. 2020;136(Suppl. 1):4-5.

42. Mateos M-V, Oriol A, Larocca A, et al. HORIZON (OP-106): An exploratory analysis of time-to-next treatment (TTNT) in patients (pts) with relapsed/refractory multiple myeloma patients (pts) with relapsed/refractory multiple myeloma
(RRMM) who received melflufen plus dexamethasone (dex). J Clin Oncol. 2020;38(Suppl. 15):e20570-e20570.

43. Edgar181. Melphalan flufenamide structure. Available at https://commons. wikimedia.org/w/index. php?curid $=55911350$ (accessed 13 July 2021).

44. Fvasconcellos. melphalan structure. Available at: https:// commons. wikimedia.org/w/index. php?curid=1448689 (accessed 13 July 2021). 